\title{
The EVER genes - the genetic etiology of carcinogenesis in epidermodysplasia verruciformis and a possible role in non-epidermodysplasia verruciformis patients
}

\author{
Agnieszka Kalińska-Bienias ${ }^{1}$, Cezary Kowalewski ${ }^{1}$, Sławomir Majewski² \\ 1Department of Dermatology and Immunodermatology, Medical University of Warsaw, Warsaw, Poland \\ ${ }^{2}$ Department of Dermatology and Venereology, Medical University of Warsaw, Warsaw, Poland
}

Adv Dermatol Allergol 2016; XXXIII (2): 75-80

DOI: 10.5114/ada.2016.59145

\begin{abstract}
In recent years, the two adjacent novel EVER1 and EVER2 genes have been identified, whose mutations are responsible for the development of epidermodysplasia verruciformis (EV). Epidermodysplasia verruciformis is a rare, autosomal recessive genodermatosis associated with increased risk of skin carcinoma. Up to now 7 mutations in the EVER1 gene and 5 mutations in the EVER2 gene have been identified only in EV. It was also determined that the EVER genes belong to a novel gene family, the transmembrane channel-like (TMC) family, and are responsible for properly functioning zinc homeostasis. These observations have given new insights into EV pathogenesis.
\end{abstract}

Key words: epidermodysplasia verruciformis, EVER genes and proteins, carcinogenesis.

\section{Introduction}

Epidermodysplasia verruciformis (EV), a rare disease characterized by verrucous cutaneous lesions and spots resembling pityriasis versicolor which may progress to squamous cell carcinomas due to abnormal susceptibility to a specific group of oncogenic EV human papillomavirus (HPV), was first described in 1922 by Lewandowsky and Lutz [1]. In 1933 Cockayne hypothesized that EV might be a congenital disease transmitted by a recessive gene [2]. Later, clinical observations of familial aggregations in 147 reported cases supported this type of inheritance, because $10 \%$ of EV families have more than one affected sibling, the proportion of siblings was $25 \%$, and the male to female ratio was close to $1: 1$ [3]. However, there was one exception - one consanguineous family in which only males developed EV and therefore $\mathrm{X}$-linked recessive inheritance was also suggested, which could point to a genetic heterogeneity of the disease [4]. In recent years two susceptibility loci for EV were mapped to chromosomal regions 17q25 (EV1) and 2p21-p24 (EV2) [5, 6], and this led to the identification of two novel genes, EVER1 and EVER2 in EV1, mutations in which play a role in development of EV [7]. The role of EVER proteins re- mains unclear. Studies conducted to date to determine the function of the EVER proteins have shown that they have an ability to participate in maintaining the zinc balance in cells [8].

The aim of this manuscript was to present the most important information and the results of the latest studies regarding EVER genes, their mutations and structures. The functions of EVER proteins and their role in HPV infections are also discussed.

\section{EVER genes and their association with epidermodysplasia verruciformis}

In 1999 Ramoz et al. identified the first susceptibility locus for EV (named EV1). In cited studies, genetic analysis included three consanguineous EV families (2 originated from Algeria marked as A1 and A2 pedigree and 1 originated from Columbia marked as C1 pedigree), in which 6 individuals were diagnosed with EV. All affected people were born from first cousin marriages. A genomewide linkage analysis using 255 highly polymorphic microsatellite markers spanning 22 autosomes was performed. It was found that the susceptibility locus for EV is located on chromosome $17 q 25$, at a distance of $1 \mathrm{cM}$

Address for correspondence: Agnieszka Kalińska-Bienias MD, PhD, Department of Dermatology and Immunodermatology, Medical University of Warsaw, 82 a Koszykowa St, 02-008 Warsaw, Poland, phone: +48 606618 564, fax: +48 225022104 , e-mail: agnieszka.kalinska@interia.pl Received: 29.03.2014, accepted: 16.11.2014. 
between the D175939 and D17S802 markers [5]. It needs to be stressed that the EV1 locus is situated in a larger region containing a locus for the susceptibility to psoriasis - PSORS2, which was previously reported [9]. It could explain the presence of EV HPV DNA in psoriatic papules in more than $90 \%$ of patients with psoriasis [10].

In 2000 the same authors mapped the second susceptibility locus for EV (EV2). Genetic analysis was carried out in two consanguineous EV families from Columbia (C2) and France (F1) comprising 7 EV patients and their families, inclusively 27 individuals. The EV2 locus was mapped to the chromosome region 2p21-p24 at an $8 \mathrm{cM}$ interval between the D2S171 and D2S2347 markers [6]. The EV1 and EV2 loci included numerous genes encoding transcription factors, proteins involved in signal transduction and numerous expressed sequence tags [11, 12]. In 1999 Kuhlenbaumer et al. reported an integrated physical and partial transcript map of region 17q25 encompassing the EV1 locus [13]. Also for the EV2 locus a map of the $1 \mathrm{cM}$ region telomeric to the D2S174 marker has been reported. It is known that this region includes 5 genes and among them is the gene encoding the centromere protein A [14]. Further studies allowed for the identification of the novel genes responsible for $\mathrm{EV}$. The analysis included two previously described Algerian (A1 and $\mathrm{A} 2$ ) and two Colombian families (C1 and C2) and one additional Algerian family (A3). It was found that the EV1 region contains the following genes: thymidine kinase 1 , synaptogyrin 2 and four novel genes named EV1Xa, $E V 1 X b, E V 1 X C$ and EV1Xd [15]. In the next stage, by RT-PCR in lymphoblastoid cell lines only EVIXC and EVIXd transcripts were obtained and assigned the symbols EVERI and EVER2 respectively. These two adjacent novel genes are in the opposite orientation from the first inframe ATG codon and are separated by $4732 \mathrm{bp}$. The presence of these mutations in both EVER1 and EVER2 genes is associated with EV [6].

\section{EVER genes - their structure, localization and correlations with the transmembrane channel- like $(T M C)$ gene family}

The EVER1 and EVER2 genes are located on chromosome $17 q 25$. The EVER1 gene consists of 20 exons and 19 introns and encodes four transcripts. Two of the transcripts containing all exons have a length of $2891 \mathrm{bp}$ and $2789 \mathrm{bp}$. The third contains 19 exons and is about $2711 \mathrm{bp}$ in length. The last one contains 12 exons and is $1838 \mathrm{bp}$ in length. The EVER2 gene consists of 16 exons and 15 introns and encodes only one transcript of the length of about 4419 bp (http://www.ensembl.org/Homo_sapiens/Transcript/). In 2003 Keresztes et al. and Kurima et al. reported that the two newly detected EVER1 and EVER2 genes were identical to the TMC6 and TMC8 genes, respectively, and belonged to a novel gene family, the transmembrane channel-like gene family (TMC) [16, 17].
All TMC gene families consist of 8 genes, which encode transmembrane proteins with 6 to 10 domains. They also showed that all TMC proteins contain a 120-amino acid sequence, the TMC domain $[16,17]$. The exact role of all TMC genes and their mutations is unknown. Besides TMC 6 and TMC8 genes it was found that dominant and recessive mutations in the TMC-1 gene and its murine ortholog-transmembrane cochlear expressed gene 1 (tmc-1), which is expressed in cochlear hair cells of the inner ear, cause hearing loss [18].

\section{Mutations and polymorphisms of EVER genes}

Mutations of the EVER1 and EVER2 genes were found in 31 (75.6\%) of $41 \mathrm{EV}$ patients in the course of a collaborative study [19]. The fact that no mutations were detected in $25 \%$ of the EV patients indicates the genetic heterogeneity of the disease and may suggest not yet identified genes responsible for the development of the disease in some cases. So far in the literature there have been reported seven in EVER1 and five in EVER2 mutations resulting from several mechanisms: nonsense mutations, single nucleotide mutations, splice site mutation, or deletion of exons (Table 1). In a group of Polish EV patients two mutations in the EVER2 gene and lack of any mutations in the EVER1 gene have been identified. The first mutation was transversion $G>T$ at nucleotide position 150 within intron 4. It has been assumed that this mutation (named IVS4-1G>T; T150fsX3) is characteristic for the Polish population of patients with EV. The second mutation leads to the deletion of nucleotide $T$ at position 705 within exon 8 (del705T, G235fsX47) (Majewski et al., data not shown). Besides mutations of EVER genes several polymorphisms have also been detected in EV. In the literature two polymorphisms of EVER genes registered in the dbSNP database in NCBI (rs7208422 and rs12452890) and one newly found (917 c. 457C $\rightarrow$ T) have been demonstrated [20-22].

\section{EVER proteins - their location and structure}

The full length transcripts of the EVER1 gene encode two polypeptide chains consisting of 805 amino acids. In alternative splice events there are synthesized two isoforms consisting of two smaller proteins of length 384 and 454 amino acids (www.ensembl.org/Homo_sapiens/ Gene). EVER1 protein is an integral membrane protein with ten domains (www.cbs.dtu.dk/services/tmhmm-2.0), two leucine-zipper motifs and two putative glycosylation sites (www.emboss.sourceforge.net/). The terminal regions of EVER1 protein are located lumenally to the cell membrane (www.uniprot.org/help/uniprot). The EVER2 gene contains one transcript of length 4419 bp, which encodes one protein of 726 amino acids. The EVER2 protein is also an integral membrane protein, which contains eight domains, three leucine-zipper motifs and two pu- 
Table 1. Mutations of EVER1 and EVER2 genes

\begin{tabular}{|c|c|c|c|c|}
\hline Mutation of EVER1 gene & Position (cDNA) & Exon & Intron & Reference \\
\hline 1. Nonsense & $220 C>T$ & 4 & - & Aochi et al., 2007 [41] \\
\hline 2. Nonsense & $280 C>T$ & 5 & - & Ramoz et al., 2002 [15] \\
\hline 3. Nonsense & $744 C>A$ & 8 & - & Tate et al., 2004 [42] \\
\hline 4. Splice-site & IVS8-2 A>T & - & 8 & Tate et al., 2004 [42] \\
\hline 5. Nonsense & 916insert CATGT & 9 & - & Zuo et al., 2006 [22] \\
\hline 6. Frameshift & 968 delT & 9 & - & Gober et al., 2008 [43] \\
\hline 7. Nonsense & $1726 \mathrm{G}>T$ & 14 & - & Ramoz et al., 2002 [15] \\
\hline Mutation of EVER2 gene & Position (cDNA) & Exon & Intron & Reference \\
\hline 1. Nonsense & $188 \mathrm{G}>\mathrm{A}$ & 3 & - & Rady et al., 2007 [44] \\
\hline 2. Fameshift & 561_583del & 6 & - & Berthelot et al., 2007 [45] \\
\hline 3. Nonsense & $568 C>T$ & 6 & - & Sun et al., 2005 [46] \\
\hline 4. Frameshift & 754 delT & 8 & - & Ramoz et al., 2002 [15] \\
\hline 5. Nonsense & $1084 G>T$ & 9 & - & Ramoz et al., 2002 [15] \\
\hline
\end{tabular}

tative glycosylation sites. Terminal regions of the EVER2 protein are located on the cytoplasmic side of the cell as distinct from EVER1. EVER1 and EVER2 proteins are 28.3\% identical in their features, and less conserved sections are present in their amino and carboxyl termini. The proteins are expressed in the cytoplasm and co-localized with calnexin, which is an integral membrane protein present in the endoplasmic reticulum [15]. The EVER genes are transcribed in keratinocytes, CD4+ and CD8+ lymphocytes, B lymphocytes and NK cells. It has also been shown that EVER1 proteins are present in endothelial cells, CD $33+$ myeloid cells and dendritic cells [23]. It needs to be stressed that in lymphoid cells the expression of EVER1 and EVER2 is higher than in the skin [24].

\section{The role of EVER proteins in the immune system}

The characteristic phenomenon in EV is impaired cell-mediated immunity leading to the persistence of established EV HPV infections and to the malignant transformation of some of the lesions [24]. In several studies a decreased T-cell count, defective T-cell proliferation in response to phytohemagglutinin ( $\mathrm{PHA}$ ) and cutaneous anergy to a variety of antigens have been reported. It is known that patients with EV overproduce tumor necrosis factor $\alpha$ (TNF- $\alpha$ ) in lesions and have a defect in AP-1 signaling in keratinocytes which are controlled by TNF receptor 1 (TNFR-1) [25].The burning question is what might be the specific role of EVER genes in creating immune responses and the consequence of the mutations of EVER genes for the function of lymphocytes. It has been observed that EVER genes are transcribed not only in the human skin but also in CD4+ and CD8+ T lymphocytes, $B$ lymphocytes and NK cells at high levels. The expression in immune system cells could point to their involvement in the immune response [24]. It is also speculated that the deficiency of EVER proteins in T-cells may contribute to susceptibility to HPV infections due to impairment of the immune response. Indeed the recent study carried out on circulating lymphocyte populations in three adult EV patients sharing the same EVER2 mutation (T150fsX3) showed mild T-cell abnormalities. The researchers observed a significant increase of memory CD4+ and effector memory CD8+ T cells, a bias of the TCR $V \alpha \beta$ and $V \gamma \delta$ repertoires and an increase of skin-homing CD4+ T-cell subsets. The count of CD4+ and CD8+ T cells and the proliferative capacity in response to anti-CD3 stimulation were normal [25]. Another study showed that activated CD4+ and CD8+ lymphocytes (via the TCR receptor) decreased expression of EVER proteins with accompanying accumulation of zinc ions in the cytoplasm [24].

\section{The role of EVER proteins in keratinocytes}

The role of proteins encoded by EVER genes was unknown until the demonstrations carried out by Lazarczyk et al., who reported that EVER proteins might be responsible for the regulation of cellular zinc balance [8]. It is known that zinc is an important ion crucial for the proper functions of numerous proteins such as enzymes [26-28], signal transduction proteins and transcription factors. The authors found that EVER proteins interact with ZnT-1 (Zn transporter) and form the complex ZnT-1/EVER, which is associated with transferring the zinc from the cytoplasm into the endoplasmic reticulum (ER) lumen, decreasing the total amount of zinc in the cytoplasm and indirectly in the nucleus. The precise role of EVER proteins within the ZnT-1/EVER complex has not been determined yet. It is hypothesized that EVER proteins may act by: (i) modulating the activity of ZnT-1 or (ii) serving as zinc transporters themselves. It has been determined that a mutation in one of the EVER genes (regardless of 
A

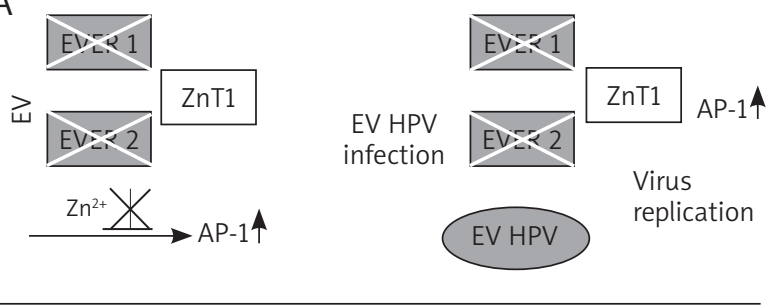

B

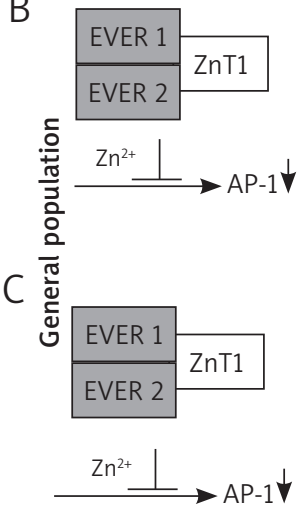

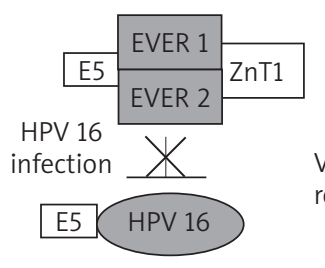

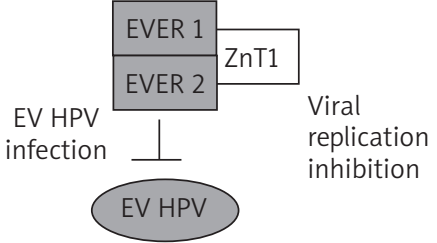

Figure 1. Mechanism of action of EVER proteins in keratinocytes: A - infection of keratinocytes by genital HPV (HPV16) leads to synthesis of $\mathrm{E} 5$ protein and inhibition of the ZnT-1/ EVER complex, which allows viral replication in the general population, B - lack of synthesis of E5 protein by EV-HPV protects against infections in the general population, $\mathrm{C}$ - in epidermodysplasia verruciformis mutations of EVER genes lead to impairment of the ZnT-1/EVER complex, allowing EV HPV infections

whether EVER1 or EVER2) interrupts the function of the whole ZnT-1/EVER complex and leads to a flux in the reverse direction from ER to the cytoplasm and the nucleus [8]. The discovery of the molecular mechanism of EVER proteins in maintaining zinc homeostasis suggests that the ZnT-1/EVER complex participates in the regulation of the activity of many transcription factors. Indeed, upon further investigation it was found that the ZnT-1/EVER complex suppressed the activity of the AP-1, Elk-1 and Fos transcription factors in keratinocytes [29]. Moreover, the suppression of AP-1 by the ZnT-1/EVER complex is also determined when AP-1 is activated by other physiological triggers such as epidermal growth factor (EGF) and tumor growth factor $\alpha$ (TGF- $\alpha$ ) [30]. It seems that cellular phosphatase and kinases might be the crucial target for the ZnT-1/EVER complex and in this way reflect the phosphorylation status of numerous cellular proteins [29].

It needs to be stressed that the results of the latest studies suggest other functions of EVER proteins. Studies carried out by Gaud et al. showed the role of EVER proteins in inducing cell apoptosis. It was determined that EVER2 protein binds TRADD and promotes TNF- $\alpha$

and TRAIL-induced apoptosis. In this process the EVER2 protein interacts with the $\mathrm{N}$-terminal domain of TRADD, and impairs the recruitment of TRAF2 and RIPK1 [31].

In 2014 Vuillier et al. analyzed the role of EVER2 protein in NF-KB and JNK/AP-1 signaling pathways. Interestingly, the results showed that EVER2 loss induces constitutive JNK activation, impairs the NF-kB pathway, sustains TRAF2 ubiquitination and decreases the pool of TRAF2. Finally, the authors demonstrated that EVER2 loss induces constitutive PKCa-dependent c-jun phosphorylation. These findings may indicate that mutations of the EVER2 gene may create the way to EV HPV replication and the persistence of lesions with the potential to develop into skin cancer [32].

\section{The role of EVER proteins in HPV infections}

The role of the "EVER barrier" in EV HPV infections in $\mathrm{EV}$ and the general population also remains a mystery. It is hypothesized that properly functioning zinc homeostasis might constitute a natural protective barrier, which limits the access of zinc ions and prevents viral replications [29]. In EV, mutations of EVER genes lead to the synthesis of impaired EVER proteins and disrupt the ZnT-1/ EVER complex, leading to an increased zinc level in the cytoplasm, allowing replication of EV HPV (Figure 1 A) and unusual sensitivity to infections by EV HPV [29]. The intriguing question is in what way does the lack of properly functioning EVER proteins in EV lead to EV and skin cancers? It has been proposed that excess of zinc ions may induce c-Jun related pathways and the transcriptional activity of AP-1. It has been proved that the AP-1 factor is crucial for expression of the viral genome [33].

The next intriguing question is why in healthy people without mutations in EVER genes and with properly functioning EVER proteins the genital HPV viruses have the ability to induce various anogenital lesions, even such as cervical cancer. The answer can be proposed by two hypotheses. According to the first one, the EVER-based barrier might be highly selective and concern EV HPV, not genital HPV; and according to the second, the EVERbased barrier is equal for both EV HPV and genital types, but genital HPVs probably develop a mechanism that facilitates their elusion of this barrier. Currently the second hypothesis seems to be more likely. The breakthrough was found in the role of the viral protein E5. Interestingly, the E5 protein is expressed only by genital HPV and not by EV HPV [34]. This protein is assumed to contribute to the development of a lesion by stimulating cell division [35]. ZnT-1/EVER was found to bind the E5 protein, leading to an increase in concentration of free zinc in keratinocytes and allowing for the replication of genital HPV (Figure $1 \mathrm{~B}$ ). In non-EV patients the lack of E5 protects against EV HPV infections (Figure 1 C) [29]. 


\section{The role of polymorphism of EVER genes in carcinogenesis}

So far in the literature there have not been any reported mutations of the EVER genes in any disorders other than EV. It needs to be stressed that there is an available report showing a link between polymorphism in the EVER genes and carcinogenesis. Patel et al. [36] showed a link between the genetic variation in the EVER2 gene and an elevated risk of squamous cell carcinoma (SCC). This study was based on the hypothesis that EVER genes, mutations of which play a key role in skin cancers in EV, may also be impaired in carcinogenesis in non-EV patients. It is also known that EV HPV DNA is detected in a high percentage of actinic keratosis (AK) (85\%) and SCC (45\%) cases [37]. It needs to be stressed that only $30-40 \%$ of EV patients develop non-carcinoma skin cancers. The clinical phenotype of EV is dependent on viral and immunological features that characterize EV patients. It could also point to the possible genetic heterogeneity of the disease [7]. Patel et al. showed that genotype TT of polymorphism rs7208422 (c.917A $\rightarrow$ T, p.N306l) in EVER2 is related to a $70 \%$ increase in the risk of SCC compared to the controls $(\mathrm{OR}=1.7 ; 95 \% \mathrm{Cl}=1.1-2.7 ; p=0.01)$. It has also been associated with seropositivity for b-HPV5 and -8, and SCC [36]. In our latest studies we also found a possible association between AK and rs7208422 TT (frequency of TT in AK was $38.5 \%$ and $26.3 \%$ in the controls, $\mathrm{OR}=1.75, p=0.056$ for recessive model of inheritance). This study could point to the potential role of polymorphism rs7208422 (c.917A $\rightarrow$ T, p.N306l) of the EVER2 gene in AK [38, article in press]. The exact mechanism of this polymorphism in carcinogenesis remains unknown, but in a recent study Gaud et al. demonstrated that the skin cancer-associated EVER2 3061 protein coded by $T$ alleles results in impaired TRADD-EVER2 interaction with lower levels of TNF- $\alpha$ apoptosis [31]. The concept of the role of polymorphisms of the EVER genes in carcinogenesis was also analyzed in cervical cancer, in which experimental and epidemiological studies have demonstrated, similarly to EV, the role of oncogenic HPV in its development. Up to now there are two publications available which assess polymorphisms of the EVER genes in this disease. Wang et al. found that some regions of the EVER genes are significantly correlated with the presence of cervical intraepithelial neoplasia III [39]. Studies carried out by Castro et al. showed a strong association between rs2290907 and rs16970849 in EVER genes and cervical cancers [40].

\section{Summary}

The discovery of the role of the EVER1 and EVER2 genes has led to better understanding of processes of skin carcinogenesis. Therefore, further research evaluating the relationships between the EVER genes, the mechanism of action of EVER protein and HPV is required to fully elucidate skin carcinogenesis.

\section{Conflict of interest}

The authors declare no conflict of interest.

\section{References}

1. Lewandowsky F, Lutz W. Ein Fall einer bisher nicht beschriebenen Hauterkrankung (Epidermodysplasia verruciformis). Arch Dermatol Syphilol 1922; 141: 193-203.

2. Cockayne EA. Inherited abnormalities of the skin and its appendages. Oxford University Press, London 1933.

3. Lutzner MA. Epidermodysplasia verruciformis. An autosomal recessive disease characterized by viral warts and skin cancer. A model for viral oncogenesis. Bull Cancer 1978; 65: 169-82.

4. Androphy EJ, Dvoretzky I, Lowy DR. X-linked inheritance of epidermodysplasia verruciformis. Genetic and virologic studies of a kindred. Arch Dermatol 1985; 121: 864-8.

5. Ramoz N, Rueda LA, Bouadjar B, et al. A susceptibility locus for epidermodysplasia verruciformis, an abnormal predisposition to infection with the oncogenic human papillomavirus type 5, maps to chromosome 17qter in a region containing a psoriasis locus. J Invest Dermatol 1999; 112: 259-63.

6. Ramoz N, Taieb A, Rueda LA, et al. Evidence for a nonallelic heterogeneity of epidermodysplasia verruciformis with two susceptibility loci mapped to chromosome regions 2p21-p24 and 17q25. J Invest Dermatol 2000; 114: 1148-53.

7. Orth G. Host defenses against human papillomaviruses: lessons from epidermodysplasia verruciformis. Curr Top Microbiol Immunol 2008; 321: 59-83.

8. Lazarczyk M, Pons C, Mendoza JA, et al. Regulation of cellular zinc balance as a potential mechanism of EVER-mediated protection against pathogenesis by cutaneous oncogenic human papillomaviruses. J Exp Med 2008; 205: 35-42.

9. Nair RP, Henseler T, Jenisch S, et al. Evidence for two psoriasis susceptibility loci (HLA and $17 q$ ) and two novel candidate regions (16q and 20p) by genome-wide scan. Hum Mol Genet 1997; 6: 1349-56.

10. Majewski S, Favre M, Orth G, Jablonska S. Is human papillomavirus type 5 the putative autoantigen involved in psoriasis? J Invest Dermatol 1998; 111: 541-2.

11. Deloukas P, Schuler GD, Gyapay G, et al. A physical map of 30,000 human genes. Science 1998; 282: 744-6.

12. Plummer SJ, Simmons JA, Adams L, Casey G. Mapping of 228 ESTs and 26 genes into an integrated physical and genetic map of human chromosome 17. Genomics 1997; 45: 140-6.

13. Kuhlenbaumer G, Schirmacher A, Meuleman J, et al. A sequence-ready BAC/PAC contig and partial transcript map of approximately $1.5 \mathrm{Mb}$ in human chromosome $17 q 25 \mathrm{com}$ prising multiple disease genes. Genomics 1999; 62: 242-50.

14. Yasunaga S, Grati M, Cohen-Salmon M, et al. A mutation in OTOF, encoding otoferlin, a FER-1-like protein, causes DFNB9, a nonsyndromic form of deafness. Nat Genet 1999; 21: 363-9.

15. Ramoz N, Rueda LA, Bouadjar B, et al. Mutations in two adjacent novel genes are associated with epidermodysplasia verruciformis. Nat Genet 2002; 32: 579-81.

16. Keresztes G, Mutai H, Heller S. TMC and EVER genes belong to a larger novel family, the TMC gene family encoding transmembrane proteins. BMC Genomics 2003; 4: 24.

17. Kurima K, Yang Y, Sorber K, Griffith AJ. Characterization of the transmembrane channel-like (TMC) gene family: functional clues from hearing loss and epidermodysplasia verruciformis. Genomics 2003; 82: 300-8.

18. Kurima K, Peters LM, Yang Y, et al. Dominant and recessive deafness caused by mutations of a novel gene, TMC1, re- 
quired for cochlear hair-cell function. Nat Genet 2002; 30: 277-84.

19. Orth G. Genetics of epidermodysplasia verruciformis: in sights into host defense against papillomaviruses. Semin Immunol 2006; 18: 362-74.

20.Arnold AW, Burger B, Kump E, et al. Homozygosity for the c.917A-->T (p.N306l) polymorphism in the EVER2/TMC8 gene of two sisters with epidermodysplasia verruciformis Lewandowsky-Lutz originally described by Wilhelm Lutz. Dermatology 2011; 222: 81-6.

21. Hohenstein E, Rady PL, Hergersberg M, et al. Epidermodysplasia verruciformis in a HIV-positive patient homozygous for the C917A-->T polymorphism in the TMC8/EVER2 gene. Dermatology 2009; 218: 114-8.

22.Zuo YG, Ma D, Zhang Y, et al. Identification of a novel mutation and a genetic polymorphism of EVER1 gene in two families with epidermodysplasia verruciformis. J Dermatol Sci 2006; 44: 153-9.

23. Su Al, Wiltshire T, Batalov S, et al. A gene atlas of the mouse and human protein-encoding transcriptomes. Proc Natl Acad Sci USA 2004; 101: 6062-7.

24. Lazarczyk M, Dalard C, Hayder M, et al. EVER proteins, key elements of the natural anti-human papillomavirus barrier, are regulated upon T-cell activation. PLoS One 2012; 7: e39995.

25. Crequer A, Picard C, Pedergnana V, et al. EVER2 deficiency is associated with mild T-cell abnormalities. J Clin Immunol 2013; 33: 14-21.

26. Eide DJ. Zinc transporters and the cellular trafficking of zinc. Biochim Biophys Acta 2006; 1763: 711-22.

27. Sekler I, Sensi SL, Hershfinkel M, Silverman WF. Mechanism and regulation of cellular zinc transport. Mol Med 2007; 13: 337-43.

28. Yamasaki S, Sakata-Sogawa K, Hasegawa A, et al. Zinc is a novel intracellular second messenger. J Cell Biol 2007; 177: 637-45.

29. Lazarczyk M, Favre M. Role of Zn2+ ions in host-virus interactions. J Virol 2008; 82: 11486-94.

30. Lazarczyk M, Cassonnet P, Pons C, et al. The EVER proteins as a natural barrier against papillomaviruses: a new insight into the pathogenesis of human papillomavirus infections. Microbiol Mol Biol Rev 2009; 73: 348-70.

31. Gaud G, Guillemot D, Jacob Y, et al. EVER2 protein binds TRADD to promote TNF-alpha-induced apoptosis. Cell Death Dis 2013; 4: e499.

32. Vuillier F, Gaud G, Guillemot D, et al. Loss of the HPV-infection resistance EVER2 protein impairs NF-kappaB signaling pathways in keratinocytes. PLoS One 2014; 9: e89479.

33. Eom SJ, Kim EY, Lee JE, et al. Zn(2+) induces stimulation of the c-Jun N-terminal kinase signaling pathway through phosphoinositide 3-Kinase. Mol Pharmacol 2001; 59: 981-6.

34. Nonnenmacher M, Salmon J, Jacob Y, et al. Cottontail rabbit papillomavirus E8 protein is essential for wart formation and provides new insights into viral pathogenesis. J Virol 2006; 80: 4890-900.

35. Genther Williams SM, Disbrow GL, et al. Requirement of epidermal growth factor receptor for hyperplasia induced by E5, a high-risk human papillomavirus oncogene. Cancer Res 2005; 65: 6534-42.

36. Patel AS, Karagas MR, Pawlita M, et al. Cutaneous human papillomavirus infection, the EVER2 gene and incidence of squamous cell carcinoma: a case-control study. Int J Cancer 2008; 122: 2377-9.

37. Pfister H, Fuchs PG, Majewski S, et al. High prevalence of epidermodysplasia verruciformis-associated human papillo- mavirus DNA in actinic keratoses of the immunocompetent population. Arch Dermatol Res 2003; 295: 273-9.

38. Kalinska-Bienias A, Kostrzewa G, Malejczyk M, et al. Possible association between actinic keratosis and polymorphism rs7208422 (c.917A $\rightarrow$ T, p.N306l) of EVER2 gene in non-EV patients. Clin Exp Dermatol 2015; 40: 318-23.

39. Wang SS, Gonzalez P, Yu K, et al. Common genetic variants and risk for HPV persistence and progression to cervical cancer. PLoS One 2010; 5: e8667.

40.Castro FA, Ivansson EL, Schmitt M, et al. Contribution of TMC6 and TMC8 (EVER1 and EVER2) variants to cervical cancer susceptibility. Int J Cancer 2012; 130: 349-55.

41. Aochi S, Nakanishi G, Suzuki N, et al. A novel homozygous mutation of the EVER1/TMC6 gene in a Japanese patient with epidermodysplasia verruciformis. Br J Dermatol 2007; 157: 1265-6.

42. Tate G, Suzuki T, Kishimoto K, Mitsuya T. Novel mutations of EVER1/TMC6 gene in a Japanese patient with epidermodysplasia verruciformis. J Hum Genet 2004; 49: 223-5.

43. Gober MD, Fishelevich R, Zhao Y, et al. Human natural killer $T$ cells infiltrate into the skin at elicitation sites of allergic contact dermatitis. J Invest Dermatol 2008; 128: 1460-9.

44. Rady PL, De Oliveira WR, He Q, et al. Novel homozygous nonsense TMC8 mutation detected in patients with epidermodysplasia verruciformis from a Brazilian family. Br J Dermatol 2007; 157: 831-3.

45. Berthelot C, Dickerson MC, Rady P, et al. Treatment of a patient with epidermodysplasia verruciformis carrying a novel EVER2 mutation with imiquimod. J Am Acad Dermatol 2007; 56: 882-6.

46.Sun XK, Chen JF, XU AE. A homozygous nonsense mutation in the EVER2 gene leads to epidermodysplasia verruciformis. Clin Exp Dermatol 2005; 30: 573-4. 